\title{
Why the enteric nervous system is important to clinicians
}

\author{
M A Kamm
}

The enteric nervous system (ENS) is central to normal gut function and is involved in most, if not all, disorders of the luminal gastrointestinal tract. The primary pathology of oesophageal and gastrointestinal disorders can lie in the ENS, in adjacent structures such as enteric smooth muscle and gut epithelium, or in extrinsic nerves (local, spinal, and cerebral) controlling gut function. Even if the primary pathology lies in another part of the gut, such as in the mucosa, or outside the gastrointestinal tract, such as in the extrinsic neural pathways, the ENS still serves as the effector neural controller, leading to a disturbance in gut function and generation of symptoms.

The ENS, therefore, serves as a useful therapeutic target for disorders in which it is the source of dysfunction and also for disorders in which it is the effector organ. For example, in treating distal ulcerative colitis, enemas containing the neurally active membrane stabiliser lignocaine have been shown to be effective, suggesting possible involvement of neural processes in the inflammatory process. In patients with irritable bowel syndrome and diarrhoea, there is substantial evidence that the primary disorder is one of cerebral regulation of gut function, mediated through autonomic nerve pathways. Yet the use of opioid analogues, which act on enteric neurones, is invaluable to diminish large bowel motor and secretory function. In patients with spinal cord injury, the ENS is intact, but constipation is common. Treatment with gut mucosal mechanical stimulation, luminal laxatives, or systemically active drugs, all of which can act on enteric nerves, results in increased peristalsis and restoration towards normal gut function.

The ultimate goal in managing patients with gastrointestinal disorders is to relieve symptoms and thereby improve quality of life. Each of the main gastrointestinal symptoms, whether derived from dysfunction in the gut or outside the gut, serves as a potential focus for therapy.

Most disorders inevitably involve disturbances of motor, sensory, and secretory function. The tendency to believe that just one of these factors is responsible for a particular disorder is naïve, and ignores the complex interrelationship of the control of these processes in enteric system organisation.

Constipation is predominantly a disorder of motor function, although sensory and secretory abnormalities can be demonstrated. In patients with constipation, there is slowing of gut transit or disability in evacuating the bowel, possibly due to impaired rectal and anal coordination. Behavioural techniques have mark- edly improved the symptoms of many patients with these disorders, the mechanisms responsible possibly ranging from altered cerebral control of gut function to altered pelvic floor striated muscle function. Alternatively, patients commonly use laxatives, which stimulate luminal chemical receptors resulting in increased secretion or increased motor activity. More recently, characterisation of the neurotransmitters involved in peristalsis ${ }^{1}$ has led to the development of specific compounds which may enhance peristaltic activity. Serotonin is centrally involved in mediating peristaltic function elicited by either stretching of the gut wall or luminal chemical stimulation. Enterochromaffin cells secrete serotonin, which acts on 5-hydroxytryptamine $(5-\mathrm{HT})_{4}$ receptors. Integrated neuronal activity in both an orad and caudal direction then results in coordinated proximal smooth muscle contraction, distal circular muscle relaxation, and longitudinal muscle contraction. Selective $5-\mathrm{HT}_{4}$ agonists appear promising in treating constipation, at least in part by hastening gut transit. ${ }^{2}$ Other neurotransmitters involved in this process may serve as useful targets in future. Gut derived circulating hormones may also be important targets in these disorders, such as inhibition of somatostatin.

In patients with irritable bowel syndrome and predominant pain and bloating, disorders of visceral sensitivity can be demonstrated. Although this is likely to be centrally mediated, peripheral sensory modification at the enteric or spinal level may be an effective therapy. Although experimental modification of visceral sensation can be achieved using drugs such as kappa opioid agonists and octreotide (a somatostatin analogue), clinical studies have been disappointing.

Vomiting and disorders of gastric emptying have been successfully targeted using $5-\mathrm{HT}_{3}$ antagonists and $5-\mathrm{HT}_{4}$ agonists, as well as via other prokinetic pathways using motilin agonists. ${ }^{3}$

Loperamide is an example of an effective compound for targeting of secretory and motility functions. This mu opioid analogue effectively diminishes both of these activities, has minimal central effects due to poor penetration of the blood-brain barrier, and is relatively free of side effects.

More than one target is often available to modify the same gut symptoms. The synthetic somatostatin analogue octreotide serves as an excellent model of the possible benefit from specific modification of a single enteric (and

Abbreviations used in this paper: $5-\mathrm{HT}$,

5-hydroxytryptamine; ENS, enteric nervous system.
Correspondence to: Professor M A Kam m.kamm@ic.ac.uk 
possibly extraintestinal) receptor. ${ }^{4}$ Octreotide has a marked effect on visceral blood flow, and inhibits gastrointestinal motor and secretory function. It has found an important role in the diagnosis and management of hormone secreting neuroendocrine tumours, in managing bleeding varices, gastrointestinal fistulas, and in the control of diarrhoea related to short bowel syndrome, ileostomy, idiopathic secretory diarrhoea, diabetic diarrhoea, and the diarrhoea associated with AIDS.

Certain disease situations provide a special possibility to explore in detail the possible role of specific gut hormones. For example, short bowel patients (as a result of previous resections) with a jejunostomy have large volume stomal outputs, which may be due in part to rapid gastric emptying of liquid. Short bowel patients with a preserved colon do not have such a high stool output and gastric emptying of liquid is normal. Examination of these two groups of patients has shown that those with a colon have high fasting peptide YY levels and have a normal postprandial rise, while those without a colon have a low fasting value and a reduced postprandial rise. Low peptide YY in short bowel patients may therefore cause rapid gastric emptying of liquids. High values in patients with a retained colon may slow gastric emptying and contribute to the "colonic brake". ${ }^{5}$

For clinicians, treatment of gastrointestinal symptoms using specifically targeted drugs has markedly improved clinical care. Further elucidation of normal and disease mechanisms promises to bring further rewards.

1 Grider JR, Foxx-Orenstein AE, Jin J-G. 5-Hydroxytryptamine 4 receptor agonists initiate the peristaltic reflex in human, rat and guinea pig intestine. Gastroenterology 1998;115:270-80.

2 Emmanuel AV, Kamm MA, Roy AJ, et al. Effect of a novel prokinetic drug, R093877, on gastrointestinal transit in prokinetic drug, R093877, on gastroint

3 Janssens J, Peeters TL, Vantrappen G, et al. Improvement of gastric emptying in diabetic gastroparesis by erythromycin. N Engl f Med 1990;322:1028-31.

4 Schulkes A, Wilson JS. Somatostatin in gastroenterology. BMF 1994;308:1381-2.

5 Nightingale JMO, Kamm MA, van der Sijp JRM, et al. Gastrointestinal hormones in short bowel syndrome. Peptide YY may be the "colonic brake" to gastric emptying. Gut 1996;39:267-72. 